\title{
Surfactants in semiconductor heteroepitaxy: Thermodynamics and/or kinetics?
}

\author{
Ivan Markov* \\ Institute of Physical Chemistry, Bulgarian Academy of Sciences, 1113 Sofia, Bulgaria
}

\begin{abstract}
The effect of surfactants on the thermodynamics and kinetics of semiconductor heteroepitaxy is briefly discussed. It is argued that the way the surfactants suppress the thermodynamic driving force for 3D islanding depends on the mechanism of exchange of overlayer and surfactant atoms. If the overlayer atoms occupy bulk-like positions provided by the outgoing surfactant dimers, as is the case of $\mathrm{Ge} / \mathrm{Si}(001)$, large atomic displacements are forcibly inhibited, and the wetting of the overlayer by the substrate becomes nearly complete. This complete wetting of the overlayer by the substrate means a zero thermodynamic driving force for 3D islanding. Once the thermodynamics do not require $3 \mathrm{D}$ islanding a change of the growth mode with the temperature should not be observed. Thus, the temperature change of the growth mode appears as an indication for the primary role of the kinetics, as in the case of $\mathrm{Ge} / \mathrm{Si}(111)$.
\end{abstract}




\section{INTRODUCTION}

The presence of surface active species (surfactants) on the surface of growing crystals changes dramatically the mode of growth, the rate of nucleation, the transition from step flow to two-dimensional (2D) nucleation, the

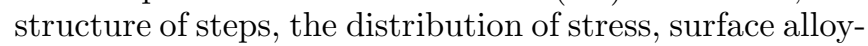
ing, the concentration of defects, etc. 6 The major effect is the suppression of the three-dimensional (3D) islanding so that smooth films suitable for fabrication of microelectronic devices could be produced. Although the initial attempts to understand the effect of the surfactants on the mode of growth were based on thermodynamic grounds, there is still a debate in the literature between the adherents of the thermodynamic and the kinetic views. That is why we will make an attempt to consider both the thermodynamic and the kinetic aspects of the effect of surfactants on the thin film growth mode. We will do that in the most general way without accounting for the particular properties of the materials. We note only that in the case of semiconductor growth a complete monolayer of the surfactant is needed 3

\section{THERMODYNAMICS}

If we want to change the direction of a process we have to change the sign of the thermodynamic force that drives it. If we want to prevent a particular process to take place we have to suppress the corresponding thermodynamic driving force making it as close to zero as possible. The thermodynamic driving force which determines the occurrence of one or another mechanism of epitaxial growth is the difference $\Delta \mu=\mu(n)-\mu_{3 D}^{0}$ of the chemical potential, $\mu(n)$, of the overlayer which depends on the film thickness measured in number $n$ of monolayers counted from the interface, and the chemical potential, $\mu_{3 D}^{0}$, of the bulk 3D crystal. 4 国 The thickness dependence of the film chemical potential $\mu(n)$ originates mostly from the thickness distribution of the strain due to the lattice misfit, but the interaction between the deposit and the substrate, which rapidly decreases with the distance from the interface and could be neglected be yond several monolayers should be also accounted for 4 . If we deposit a crystal $A$ on the surface of a crystal $B$ in absence of a surfactant the thermodynamic driving forfe can be written in terms of surface energies $\sigma_{A}$ and $\sigma_{B}$ a

$$
\Delta \mu=a^{2}\left[\sigma_{A}+\sigma_{A B}(n)-\sigma_{B}\right]
$$

where $a^{2}$ is the area occupied by an atom, and $\sigma_{A B}(n)$ is the interfacial energy which includes in itself the misfit strain energy and the attenuation of the energetic influence of the substrate. 6 In fact, this is the familiar 3- $\sigma$ criterion of Bauer.t

We can write the above expression in terms of interatomic energies

$$
\mu(n)=\mu_{3 D}^{0}+\left(E_{A A}-E_{A B}\right)=\mu_{3 D}^{0}+E_{A A} \Phi
$$

where $E_{A A}$ and $E_{A B}$ are the energies per atom to disjoin a half-crystal $A$ from a like half-crystal $A$ and an unlike half-crystal $B$. It is in fact the adhesion energy $E_{A B}$ which includes in itself the thickness distribution of the strain energy. In the above equation $\Phi=1-E_{A B} / E_{A A}$ is the so called adhesion parameter which accounts for the wetting of the overgrowth by the substrate. This is the same parameter which enters the work of formation of nuclei on a foreign substrate in the classical nucleation theory.

Equations (11) and (2) are identical. Eq. (1) can be readily obtained from (2) by using the definition of surface free energy and the relation of Dupré.6 The essential physics, however, is in Eq. (2) as the surface energies are derivatives of the interatomic forces. It shows that the thermodynamic driving force for occurrence of one or another mode of growth is the relative adhesion/cohesion difference, or in other words, the wetting. The incomplete wetting, $0<\Phi<1$, is the driving force for 3D nucleation and growth, whereas the complete wetting, $\Phi \leq 0$, is the driving force for planar growth by nucleation and growth of $2 \mathrm{D}$ monolayer height nuclei.

When $0<\Phi<1 \mu(n)>\mu_{3 D}^{0}$, and 3D islands are formed on top of the substrate surface from the very beginning of deposition. This is the well known VolmerWeber (VW) growth and $\mu(n)$ goes asymptotically to $\mu_{3 D}^{0}$ from above (the curve denoted by VW in Fig. 11).

In the other extreme of complete wetting $(\Phi \leq 0)$ either layer-by-layer (Frank-van der Merwe or FM) growth at negligible lattice misfit, or a Stranski-Krastanov (SK) growth (layer-by-layer growth followed by 3D islands at a perceptible misfit, are thermodynamically favored. In the case of FM growth $\mu(n)$ goes asymptotically to $\mu_{3 D}^{0}$ from below (the curve denoted by FM in Fig. 1). The first monolayer has the lowest chemical potential owing to the strongest interaction with the substrate, and a second monolayer can form only after the completion of the first one.

The SK growth mode appears as a result of the interplay of the film - substrate bonding, strain, and surface energies. A wetting layer consisting of an integer number of monolayers is first formed by a FM mode which is driven by a negative thermodynamic driving force $\Delta \mu$ (complete wetting). Further FM growth becomes unfavorable as the strain energy accumulates linearly with film thickness. In addition, the stronger attraction from the substrate, that overcompensates the strain energy, disappears beyond several atomic diameters. On top of the wetting layer 3D islands form and grow under the influence of a positive thermodynamic driving force (incomplete wetting). If misfit dislocations (MDs) are introduced immediately after the completion of the wetting layer unstrained 3D islands are formed and grow on top of it. This is the classical Stranski-Krastanov growth in which the work needed to create new surfaces is overcompensated by a complete strain relaxation. It 
was found recently that initially coherent (dislocationfree) 3D islands are formed (coherent Stranski-Krastanov growth) 1113 in which the formation of a new surface is overcompensated by a gradual strain relaxation. In both cases the 3D islands and the wetting layer represent necessarily different phases separated by an interphase boundary. This boundary is determined by the displacements of the atoms belonging to the first atomic plane of the islands from the bottoms of the corresponding potential troughs of the uniformly strained wetting layer. In the classical SK grpwth the displacements are described in terms of MDs,14 whereas in the coherent SK growth the atoms that are closer to the islapds edges are displaced owing to the islands finite size 15 These displacements give rise to weaker adhesion, or in other words, to incomplete wetting. It is namely this incomplete wetting (or weaker adhesion) which appears as the thermodynamic driving force for the 3D islanding in the SK mode of growth. In other words, we can treat the SK mode as a FM mode driven by complete wetting $(\Delta \mu<0)$, followed by VW mode driven by incomplete wetting $(\Delta \mu>0)$ (the curve denoted by SK in Fig. 1).

When considering the effect of the surfactant on the mode of growth we note that the chemical potential $\mu_{3 D}^{0}$ of the infinitely large crystal does not depend on whether there are impurities adsorbed on its surface or not 16,17 This, however, is not true for sufficiently thin films. If a monolayer thick film of $A$ is covered by a monolayer of a surfactant (S) atoms the growth mode criterion (11) turns into 18

$$
\Delta \mu_{S}=a^{2}\left(\sigma_{A B}+\sigma_{S A}-\sigma_{S B}\right)
$$

where the $\sigma$ 's are the corresponding interfacial energies. This expression is equivalent to

$$
\mu_{S}(n)=\mu_{3 D}^{0}+\left(E_{A A}-E_{A B}\right)-\left(E_{S A}-E_{S B}\right)
$$

where $\mu_{S}(n)$ is the chemical potential of the film covered by $\mathrm{S}$ atoms. It is immediately seen that if $3 \mathrm{D}$ islanding is thermodynamically favored in absence of a surfactant, i.e. $E_{A A}>E_{A B}$, the inequality (四) may have the opposite sign if the adhesion of the surfactant is stronger to the overlayer rather than to the substrate and thus the third term in (4) overcompensates the second one. Then the surfactant is "good" from thermodynamic point of view if it adheres more strongly to the overlayer, thus changing the sign of the thermodynamic driving force.

This is valid, however, only for the transition from VW to FM growth as if the case of the As mediated growth of $\mathrm{Si}$ on $\mathrm{Ge}(001)$. 19 Even in this case it is valid only at zero misfit. After several monolayers of $A$ the energetic contact between the surfactant and the substrate $B$ is completely lost $\left(E_{S B} \rightarrow E_{S A}\right)$, and if the misfit is large 3D islands should appear resulting in SK mode. In systems that follow the SK growth mode where, beyond the wetting layer, $A$ is deposited on strained $A, 20$ the difference $E_{S A}-E_{S B}$ vill be nearly equal to zerp Many papers both theoretical21 24 and experimental25 30 have been devoted to the problem considering mainly the surfactant growth of $\mathrm{Ge}$ on $\mathrm{Si}(001)$. However, in most of the papers quoted above the mechanism of the exchange of the surfactant (As or $\mathrm{Sb}$ ) atoms with Ge atoms belonging to the first monolayer on the $\mathrm{Si}(001)$ surface have been studied. It is obvious, that such studies cannot explain the effect of the surfactant to suppress the 3D islanding as the first monolayer belongs to the wetting layer and it will grow in a FM mode pseudomorphous with the substrate irrespective of there is a surfactant on top or not.

Obviously, in the SK mode the surfactant should make the thermodynamic driving force for 3D islanding on top of the wetting layer equal to zero for considerable film thickness. As mentioned above in the SK mode the atoms which are in contact with the wetting layer should be displaced from their "bulk-like" positions either to make MDs or near to the islands edges. These displacements make the wetting incomplete, or which is the same, make the adhesion weaker than the cohesion $\left(E_{A B}<E_{A A}\right)$, which is the thermodynamic driving force for 3D islanding. As found in a series of papers the surfactant group- $\mathrm{V}$ atoms form dimers on $\mathrm{Si}(\mathrm{Ge})(001)$ which reside exactly where the $\mathrm{Si}(\mathrm{Ge})$ dimers should be located 25, 27.31 33 After the surfactant and overlayer dimers exchange places the latter occupy the epitaxial sites that are provided by the outgoing surfactant. The surfactant dimers on top do not permit large displacements of the overlayer atoms underneath, and in turn incomplete wetting. In other words, the surfactant atoms press the overlayer atoms into bulk-like positions and do not allow the formation of misfit dislocations. Thus, the surfactants suppress the thermodynamic driving force for $3 D$ islanding by not allowing large atomic displacements. Once the thermodynamics do not require 3D islanding a change of the growth mode with the temperature should not be observed.

\section{KINETICS}

In absence of a surfactant the atoms arriving at the crystal surface diffuse on it, join pre-existing steps or islands, or give rise to new islands. At sufficiently high temperatures the diffusivity of the atoms on the terraces is high and they reach the pre-existing steps before meeting with each other. This results in a step-flow growth. At low temperatures and in turn low diffusivity the atoms meet with each other before reaching the steps and give rise to $2 \mathrm{D}$ nuclei on the terraces. In presence of a complete monolayer of $\mathrm{S}$ atoms two new phenomena take place. First, the surfactant changes the energetics of incorporation of atoms into ascending and descending steps, and second, the overlayer atoms have to exchange places with the $\mathrm{S}$ atoms on the terraces or at the steps in order to join the crystal lattice. Both phenomena lead to a change of the diffusivity of the adatoms across the steps 
and on terraces, and in turn exert an effect on the direction of transport (upward or downward) of atoms. Moreover, the change of diffusivity on terraces affects strongly the kinetics of nucleation and growth of the islands thus stimulating either the step flow or 2D nucleation. We consider in more detail the effect of the surfactant on the kinetics of attachment and detachment of atoms to and from the steps, and the exchange-deexchange kinetics on terraces.

Whereas an atom approaching an ascending step joins it upon striking, an atom approaching a descending step has to oyercome an additional Ehrlich-Schwoebel (ES) barrier 34.35 If the ES barrier is low the overlayer grows more or less in a layer-by-layer mede. Otherwise, instabilities of different kind appear.36 Zhang and Lagally pointed out the possibility of a reduction of the ES barrier when overlayer atoms exchange sites with $\mathrm{S}$ atoms that decorate the steps, in such a way that the last barrier before descending is shifted downwards below the level of the surface diffusion barrier 37 The latter is in good agreement with first principle molecular dynamics calculations of displacement of Sb dimer at the step edge by a $\mathrm{Si}$ dimer through a push-out mechanism. $\mathrm{B}$ In addition, Markov has suggested that atoms that approach ascending steps have to overcome an extra energetic barrier owing to the necessity to displace the $\mathrm{S}$ atoms already adsorbed at the step edges. 39 These effects which are of purely kinetic origin can suppress the thermodynamic driving force for 3D islanding in heteroepitaxial growth.

When thermodynamics predict 3D islanding, $(\Delta \mu>0)$ the atoms are more strongly bound to the upper layers rather than to the lower layers. In other words the ppper layers have lower chemical potentials (Fig. 11) 4.5 This results in a gradient of the chemical potential that drives the atoms upwards-and is the driving force for the 2D3D transformation. 46 The appearance of an additional barrier at the ascending steps, and the reduction of the ES barrier at the descending steps owing to the decoration of the steps by $\mathrm{S}$ atoms, reverse the asymmetry of the attachment - detachment kinetics and thus lead to a suppression of the thermodynamic driving force for 2D$3 \mathrm{D}$ transition. A reverse gradient of adatoms of a kinetic origin appears that drives the atoms downwards. The latter results in a planar rather than a 3D growth 39,40 Thus the attachment - detachment kinetics suppress the thermodynamic driving force for 3D islanding. At high temperature the thermodynamics prevail and SK growth takes place irrespective of the presence of the surfactant. Surfactant induced FM growth occurs at lower temperatures. Note that this kinetic effect does not require a complete monolayer of the surfactant but a small amount which is sufficient to decorate the steps.

In the presence of a complete monolayer of the surfactant a new kinetic effect appears owing to the necessity of the overlayer atoms to join the crystal lattice and of the $\mathrm{S}$ atoms to float over the surface. These are the phenomena of exchange and deexchange of overlayer and $\mathrm{S}$ atoms. Zhang and Lagally 37 first assumed that an exchange process between overlayer and $\mathrm{S}$ atoms on the terraces should take place on the $\mathrm{S}$ precovered surfaces. Kandel and Kaxiras assumed later that a deexchange process is also possible and can play a significant role in epitaxial growth. 47 They calculated the values of 0.8 and $1.6 \mathrm{eV}$ for the activation energies, $E_{e x}$ and $E_{d e x}$, for exchange and deexchange processes, respectively. Ko, Chang and Yi computed through first-principles pseudopotential total-energy calculations the values of the barriers for surface diffusion, exchange and deexchange for $\mathrm{Ge}$ on $\mathrm{Ga}$, $\mathrm{As}$ and $\mathrm{Sb}$ precovered $\mathrm{Si}(111)$ surface. They found that in all cases $E_{d e x}>E_{e x}$ but the differences are notas large as the ones calculated by Kandel and Kaxiras. 48

We introduce two time constants which characterize the exchange and the deexchange processes both being normalized to the time, $t_{1}=1 / F$, necessary for deposition of a completemonolayer, $F$ being the the atom arrival frequency. 49 . 50 First, this is the mean residence time of the atoms on top of the $\mathrm{S}$ layer before exchange $\tau_{e x}=(F / \nu) \exp \left(E_{e x} / k T\right)$ where $\nu$ is the attempt frequency. Second, this is the mean residence time before deexchange of an atom embedded in (or under) the $\mathrm{S}$ layer $\tau_{d e x}=(F / \nu) \exp \left(E_{\text {dex }} / k T\right)$. Obviously, if a particular time constant is greater than unity the corresponding process will not occur. As $\tau_{d e x} \gg \tau_{e x}$ three possibilities exist. The first is $\tau_{d e x} \ll 1$. This extreme describes the reversible exchange as the adatoms have sufficient time to go back on top of the $\mathrm{S}$ layer through a deexchange process. A dynamic exchange - deexchange equilibrium is established. With the value of $E_{\text {dex }}=1.6$ $\mathrm{eV}$ calculated by Kandel and Kaxiras we find, at $600 \mathrm{~K}$, $\tau_{d e x} \approx 0.002$. Then the exchange - deexchange equilibrium is established in the very beginning of the deposition process. 40 The nucleation process takes place in a more or less disordered (because of the different size of the atoms involved) 2D phase - a monolayer consisting of mixed S and overlayer atoms. A considerable fraction of the incoming atoms remains on top of the $\mathrm{S}$ layer and diffuse fast to the pre-existing steps. The formation, growth and decay of nuclei take place through exchange and deexchange processes. The second extreme is $\tau_{e x} \ll 1<\tau_{\text {dex }}$. This is the case of irreversible exchange. The incoming atoms rapidly exchange places with $\mathrm{S}$ atoms and remain buried under the S layer. All processes of diffusion, nucleation and incorporation into islands and steps occur under (or between) the $\mathrm{S}$ atoms. The case $1<\tau_{e x} \ll \tau_{\text {dex }}$ should be excluded as it means that the $\mathrm{S}$ atoms will remain buried under the arriving overlayer atoms.

We calculate further the nucleus density, $N_{S}$, in both cases of reversible and irreversible exchange. This is extremely important because of two reasons. First, the large number of small islands will promote layer-by-layer growth owing to the existence of a critical island size for second layer nucleation. 5255 Second, higher nucleation rate leads to growth by $2 \mathrm{D}$ nuclei, whereas in the oppo- 
site case step-flow growth will take place. In all cases the expression for $N_{S}$ can be written in the form

$$
N_{S}=N_{0} \exp \left(-\frac{\chi}{i} E_{S} / k T\right)
$$

where $E_{S}$ combines all energy contributions which depend on the presence of the surfactant, 190 and

$$
N_{0}=\left(\frac{\nu}{F}\right)^{-\chi} \exp \left(\frac{\chi}{i} E_{0} / k T\right)
$$

is thenucleus density in absence of a surfactant $\left(E_{S}=\right.$ 0 ), 1990 Thus, the island density scales with the ratio $\nu / F$, the scaling exponent $\chi$ being a function of the number $i$ of atoms in the critical nucleus 41.42 In the case of low barriers for attachment of atoms to 2D nuclei, the latter "feel" on ther through the diffusion fields around them, and 41.42

$$
\chi=\frac{i}{i+2} .
$$

Kandel has recently shown by using a rate equation approach that in presence of surfactants which give rise to barriers for incorporation of atoms to the critical nucleit3

$$
\chi=\frac{2 i}{i+3} .
$$

The scaling exponent (8) has been independently derived by Markov by using a different approach. 4 He showed that it is characteristic for kinetic regime of growth where the adatom concentration between the islands is practically constant and the islands "do not feel" the presence of each other.

As has been noted in Ref. (43) the scaling exponent (7) varies with $i$ from $1 / 3$ to 1 , whereas (8) has values larger than unity already at $i>2$. Thus, one could distinguish between diffusion and kinetic regimes of growth if $\chi$ is smaller or greater than unity. Typical examples of the scaling exponent ( 8 ) are the homoepitaxy of $\mathrm{Si}$ on $\mathrm{Sn}$ precovered surface of $\mathrm{Si}(111), 45$ and of $\mathrm{Ge}$ on $\mathrm{Pb}$ precovered surface of $\mathrm{Si}(111) 46$

It is immediately seen that the exponential multiplying $N_{0}$ in Eq. (5) can be smaller or greater than unity depending on the interplay of the energies involved in $E_{S}$. If $E_{S}<0$ the surfactant will stimulate the nucleus formation $\left(N_{S} \gg N_{0}\right)$. The latter means that a greater density of smaller islands will be formed, and the film will grow by $2 \mathrm{D}$ nucleation rather than by step-flow. What is more important is that the greater density of smaller nuclei leads to layer-by-layer growth rather than to the mound formation. In the opposite case the surfactant will inhibit the nucleation and will promote the step-flow growth. In the case of reversible exchange $E_{S}$ contains the term $-i\left[\left(E_{d e x}-E_{e x}\right)-\Delta E_{s d}\right]$ where $\Delta E_{s d}=E_{s d}^{\circ}-E_{s d}$ is the the difference between the barriers for diffusion on the clean substrate, $E_{s d}^{\circ}$, and on the surfactant passivated surface, $E_{s d},\left(E_{s d}^{\circ}>E_{s d}\right)$. If $\Delta E_{s d}$ overcompensates the difference $E_{d e x}-E_{e x}$, we could expect that $E_{S}>0$, the nucleus formation will be inhibited, $\left(N_{S} \ll N_{0}\right)$, and surfactant-induced step-flow growth will take place. 49.50

In the case of irreversible exchange Eq. (5) is still valid but the barriers $E_{d e x}$ and $E_{e x}$ do not enter the energy term $E_{S}$. The largest term in $E_{S}$ is the increment of the surface diffusion barrier $-i\left(E_{s d}-E_{s d}^{\circ}\right)$ where now $E_{s d}>E_{s d}^{\circ}$ owing to the fact that the process of diffusion under the S layer is inhibited compared with that on the clean surface. It is unlikely this term to be overcompensated by the smaller positive terms, and hence, we can always expect that the surfactant will stimulate the $2 \mathrm{D}$ nucleation rather than the step-flow growth.

Finally, we discuss briefly the reasons that lead to a change of the island density. Usually the latter is attributed to the change of atoms diffusivity. However, the change the island density might be due to other reasons. The nucleation rate depends on the Gibbs free energy for nucleus formation which is in turn a function of the nucleus size. The presence of the surfactant could lead tochange of the number of atoms in the critical nucleus. 11 As a result the values of $E_{S}$ and $E_{0}$ in Eqs. (5) and (6), as well as the scaling exponent $\chi$ will change in one and the same temperature interval. In addition, the regime of growth can change from diffusion to kinetic, so that the scaling exponent (7) will be replaced by (8). In this case, the island density will increase by orders of magnitude even if $i$ remains constant.

\section{GROWTH OF GE ON SI}

We discuss in more detail a "mode" system such as $\mathrm{Ge} / \mathrm{Si}$. As pointed out by Kaxiras,57 one and the same surfactant can have qualitatively different effect depending on the crystallographic orientation of the substrate. On $\mathrm{Si}$ or $\mathrm{Ge}(001)$ the surfactants form dimers which do not break during the exchange process.21 27 On (111) surface three different geometries (substitutional, trimers, and zig-zag chpins) of the group- $\mathrm{V}$ adsorbates $(\mathrm{P}, \mathrm{As}, \mathrm{Sb})$ are possible. $\mathrm{T}$ In the first $1 \times 1$ structure one monolayer of $\mathrm{S}$ atoms is incorporated into the upper part of the (111) bilayer, whereas in the last two structures a monolayer of $\mathrm{S}$ atoms is bonded on top of a complete (111) bilayer. In addition, the growth of the two surfaces of Si or Ge differs drastically. Whereas the (001) surface grows by single monolayers, 58 the (111) surfaces grow by bilayers 59 Thus, we have completely different mechanisms of exchange of S and overlayer atoms. In the case of (001) surfaces the growth unit is a dimer so that an exchange of $\mathrm{S}$ and opprlayer dimers takes place through a single monolayer.21 27 On (111) surfaces the mechanism of exchange depends on the surface geometry of the surfactant. For example, if the surfactant geometry is substitutional we could suppose that single overlayer atoms should displace single $\mathrm{S}$ atoms from their positions in the upper monolayer that constitutes the bilayer. After the complete building of the upper monolayer atoms 
belonging to the next lower monolayer should be incorporated first in order to serve as a template for the $\mathrm{S}$ atoms in the next upper monolayer. Thus one surfactant monolayer should be displaced by a complete bilayer of the overgrowth the process taking place by exchange of single atoms. Comparing both surfaces we could speculate that on (111) surface the surfactant could not suppress successfully the thermodynamic driving force for $3 \mathrm{D}$ islanding by not allowing large atomic displacements (formation of misfit dislocations).

We consider first the growth of Ge on Si(111). Hwang, Chang and Tsong studied this system by using $\mathrm{Pb}$ as a surfactant. They established that the $\mathrm{Pb}$ atoms make a substitutional $1 \times 1$ structure on top of the Ge surface. It was found that the saturation nucleus density in the submonolaver regime scales with the deposition flux with $\chi=1.76 .46$ This clearly shows that in the $\mathrm{Pb}$ mediated epitaxy of $\mathrm{Ge}$ on $\mathrm{Si}(111)$ the scaling exponent is given by Eq. (8) and the growth proceeds in a kinetic regime. In other words, significant energy barriers appear for attachment of atoms to the critical nuclei owing to the necessity of exchange of overlayer and $\mathrm{S}$ atoms. The same scaling exponent $(\chi=1.8)$ has been obtained in the homoepitaxial growth of Si on Sn precovered surface of $\mathrm{Si}(111) 45$ after comparing the experimental data for the critical terrace width for step flow growth with the corresponding theory 49,50 Under clean conditions in the same system Voigtländer et al. obłained a scaling exponent smaller than unity $(\chi=0.85) 60$

The observations of Hwang et al. show unambiguously that $\mathrm{Pb}$ has a prominent kinetic effect on the submonolayer growth of $\mathrm{Ge}$ on $\mathrm{Si}(111) .46$ In a further study the same authors found that 3D islanding is suppressed below $470 \mathrm{~K}$. At temperatures higher than 470K 3D islanding takes place.61 This observation could be explained by the suppression of the thermodynamic driving force for 3D islanding by the attachment - detachment kinetics mentioned above. The upward surface flux is hindered whereas the downward flux is enhanced. We could conclude that the suppression of 3D islanding in Ge growth on $\mathrm{Pb}$ precovered $\mathrm{Si}(111)$ is due more to kinetic rather than to thermodynamic reasons.

Voigtländer and Zinnerstudied the growth of Ge on Si(111) mediated by Sb.62 They established the same substitutional structure of the $\mathrm{Sb}$ atoms on the surface of Ge. They found that a transition from surfactant mediated layer-by-layer growth to the equilibrium SK mode takes place above twice higher temperatures (900K) as compared with the $\mathrm{Pb}$ mediated growth. The higher temperatures could be related to stronger chemical bonding but qualitatively the surfactant effect is the same.

The system $\mathrm{Ge} / \mathrm{Si}(001)$ is the most studied one. 3 Ide has observed that after annealing at about $800 \mathrm{~K}$ for 10 min the 2D Ge islands formed in submonolayer regime on As precovered $\mathrm{Si}(001)$ did not change whereas the islands deposited under clean conditions disappeared. 30 This observation shows that $2 \mathrm{D}$ islands formed under the surfactant layer are more stable than those without $\mathrm{S}$ atoms on top of them. Antimony mediated deposition and annealing experiments carried out by Osten et al. showed that Sb prevents 3D islanding, but alsq san smooth an already islanded film.29 Katayama et al.63 found that as the coverage of the surfactant increases, the intermixing of $\mathrm{Ge}$ and $\mathrm{Si}$, as well as the nucleation and the growth of macroscopic Ge islands, are suppressed. Both observations could be explained with the forced occupation of epitaxial sites by the Ge atoms under the influence of the $\mathrm{S}$ dimers on top of them. On the other hand, Ide also established that at $800 \mathrm{~K}$ the Ge atoms reach and join the preexisting steps of $\mathrm{Si}$ (step flow growth) on the clean surface, whereas 2D islands are formed in the presence of As. 30 The latter unambiguously demonstrates the kinetic effect of the surfactant on the mode of growth by decreasing the diffusivity of the incoming atoms.

Most of the studies of surfactant mediated growth of $\mathrm{Ge} \mathrm{np}_{\mathrm{Si}} \mathrm{ON1}$ syrface are carried out at or near to

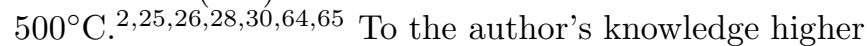
temperature deposition has been carried out by Tromp and Reuter $\left(630^{\circ} \mathrm{C}\right), 21$ and by Horn von Hoegen et al. 66 at $700^{\circ} \mathrm{C}$. No $3 \mathrm{D}$ islanding except for mound formation has been noticed on top of the wetting layer at the highest temperature used.66 A change of the growth mode with temperature as in the case of $\mathrm{Ge} / \mathrm{Si}(111)$ has not been reported so far 67 This can be interpreted as follows. The surfactant suppresses successfully the thermodynamic driving force for 3D islanding by not allowing large atomic displacements and the growth continues in a layer-by-layer mode (with the unavoidable roughness to relieve the strain) untill MDs are introduced at the interface. Once the thermodynamics do not require 3D islanding a change of the growth mode with the temperature should not be observed.

Jenkins and Srivastava carried out first principle density functional theory (DFT) calculations of the mode of growth of Ge on Sb precovered $\mathrm{Si}(001) 24$ They studied the behavior of the chemical potential of the Ge film relative to the bulk chemical potential, $\mu_{3 D}^{0}$, in absence and presence of $\mathrm{Sb}$ as a surfactant. They found that in absence of $\mathrm{Sb}$ the chemical potentials of the first three monolayers of Ge, which belong to the wetting layer, are smaller (more negative) than $\mu_{3 D}^{0}$ (complete wetting). The chemical potential of the forth monolayer becomes more positive than $\mu_{3 D}^{0}$ (incomplete wetting) which is an indication of $3 \mathrm{D}$ islanding after the first three monolayers. In presence of $\mathrm{Sb}$ the chemical potential of the first monolayer is slightly more negative that $\mu_{3 D}^{0}$ but the chemical potentials of the remaining three monolayers are equal to $\mu_{3 D}^{0}$ within the accuracy of the calculations. An error is introduced in the calculations by not allowing the Ge overlayers to relax laterally. Thus after the exhange of Ge and Sb dimers the Ge atoms occupy the bulk-like positions left by the outgoing Sb dimers and the surfactant successfully suppressed the thermodynamic driving force for 3D islanding. In fact, the surfactant replaced the curve SK by the curve FM in Fig. (11).

Similar first principle DFT calculations have been car- 
ried out by Gonzá- les-Méndez and Takeuchi for the growth of $\mathrm{Si}$ on $\mathrm{Ge}(001)$ by using As as a surfactant. 19 In absence of a surfactant $\mathrm{Si}$ grows in $\mathrm{VW}$ mode as $3 \mathrm{D}$ islands directly on the Ge surface.68 In presence of As the overlayer prefers to grow in a layer-by-layer mode due to thermodynamic reasons, the energy of the $3 \mathrm{D}$ islands being always lower than that of the complete monolayers. The deposit atoms are almost in bulk-like positions. Equally important, the authors established that As greatly reduces the $\mathrm{Si}$ and Ge intermixing. This is another indication of the thermodynamic influence of the surfactant as the alloying also requires large atomic displacements.

\section{CONCLUSION}

The presence of a third element in the system unavoidably changes the kinetics of growth. Hence, we have to decide whether the kinetics is solely responsible for suppression of the 3D islanding, or the thermodynamics plays a decisive role, with the kinetics exerting an additional effect. We argue that this question could be answered by studying the temperature dependence of the capability of the surfactant to suppress $3 \mathrm{D}$ islanding. If the kinetics play a decisive role we should expect a change of the mode of growth from layer-by-layer at lower temperature to $3 \mathrm{D}$ (SK or VW) islands at higher temperature. The physical reason is that at high temperature the system is closer to equilibrium whereas at lower temperature the kinetic effects suppress the thermodynamic driving force for 3D islanding. This is most probably the case of the system $\mathrm{Ge} / \mathrm{Si}(111)$ where one monolayer of the surfactant should be displaced by two monolayers of the overgrowth and the exchange process takes randomly place by single atoms. The S atoms do not press the overlayer atoms into bulk-like positions and formation of misfit dislocations or displacements of the edge atoms is not inhibited. We have seen that the growth is accompanied by typical kinetic phenomena as the kinetic regime of growth $(\chi>1)$. On the contrary, the system $\mathrm{Ge} / \mathrm{Si}(001)$ demonstrates the decisive role of the thermodynamics. The kinetics play an auxiliary role in the same direction. The physical reason for suppression of the $3 \mathrm{D}$ islanding is that a monolayer of the surfactant is displaced by a monolayer of the film during growth, and the exchange process occurs by dimers rather than by single atoms. As a result the overlayer atoms occupy nearly bulk-like positions provided by the outgoing surfactant dimers. Large atomic displacements are forcibly prohibited which in turn leads to complete wetting and a zero thermodynamic driving force for $3 \mathrm{D}$ islanding. It is thus clear that the third element in the system does not act as a surfactant at all.

We conclude that the relative weight of the effect of the third element on the thermodynamics and kinetics in semiconductor heteroepitaxy depends mostly on the mechanism of exchange of overlayer and surfactant atoms. Undoubtedly the third element changes the surface energies but it is the change of the relative adhesion of the overlayer to the substrate that is crucial from thermodynamic point of view.

\section{ACKNOWLEDGMENTS}

The author is greatly indebted to M. Paunov for the fruitfull discussion and the critical reading of the manuscript.

* Electronic mail address: imarkov@ipc.bas.bg (Ivan Markov); Fax: (+359) 29712688.

${ }^{1}$ M. Zinke-Allmang, Thin Solid Films 346, 1 (1999).

${ }^{2}$ M. Copel, M. C. Reuter, E. Kaxiras, and R. M. Tromp, Phys. Rev. Lett. 63, 632 (1989).

${ }^{3}$ D. Kandel and E. Kaxiras, Solid State Phys. 54, 219 (2000).

${ }^{4}$ S. Stoyanov, Surf. Sci. 172, 198 (1986).

${ }^{5}$ I. Markov and S. Stoyanov, Contemp. Phys. 28, 267 (1987).

${ }^{6}$ I. Markov, Crystal Growth for Beginners, World Scientific, 1995, p. 389.

${ }^{7}$ E. Bauer, Z. Kristallographie, 110, 372 (1958).

${ }^{8}$ R. Kaischew, Commun. Bulg. Acad. Sci. (Phys.) 1, 100 (1950).

${ }^{9}$ R. Kern, G. LeLay, and J. J. Metois, in Current Topics in Materials Science, vol. 3, ed. by E. Kaldis, (North-Holland, 1979).

${ }^{10}$ M. H. Grabow and G. H. Gilmer, Surf. Sci. 194, 333 (1988).

${ }^{11}$ D. J. Eaglesham and M. Cerullo, Phys. Rev. Lett. 64, 1943 (1990).

12 D. Leonard, M. Krishnamurty, C. M. Reaves, S. P. Denbaars, and P. M. Petroff, Appl. Phys. Lett. 63, 3203 (1993).

${ }^{13}$ Vinh Le Thanh, P. Boucaud, D. Débarre, Y. Zheng, D. Bouchier, and J.-M. Lourtioz, Phys. Rev. B 58, 13115 (1998).

14 J. W. Matthews, D. C. Jackson, and A. Chambers, Thin Solid Films 29, 129 (1975).

${ }^{15}$ E. Korutcheva, A. M. Turiel, and I. Markov, Phys. Rev. B 61, 16890 (2000).

${ }^{16}$ G. Bliznakov, Commun. Bulg. Acad. Sci. (phys. ser.) 3, 23 (1953).

17 N. Pangarov, Electrochim. Acta 28, 763 (1983).

${ }^{18}$ M. Paunov, Cryst. Res. Technol. 33, 165 (1998).

${ }^{19}$ M. E. González-Méndez and N. Takeuchi, Surf. Sci. 441, L897 (1999).

${ }^{20}$ C. W. Snyder and B. G. Orr, Phys. Rev. Lett. 70, 1030 (1993).

${ }^{21}$ R. M. Tromp and M. C. Reuter, Phys. Rev. Lett. 68, 954 (1992).

22 B. D. Yu and A. Oshiyama, Phys. Rev. Lett. 72, 3190 (1994). 
${ }^{23}$ M. Jiang, X. Zhou, B. Li, and P. Cao, Phys. Rev. B 60, 8171 (1999).

${ }^{24}$ S. J. Jenkins and G. P. Srivastava, Surf. Sci. 398, L308 (1998).

${ }^{25}$ R. Cao, X. Yang, J. Terry, and P. Pianetta, Appl. Phys. Lett. 61, 2347 (1992).

${ }^{26}$ B. D. Yu, T. Ide, and A. Oshiyama, Phys. Rev. B 50, 14631 (1994)

${ }^{27}$ M. A. Boshart, A. A. Bailes III, and L. E. Seiberling, Surf. Sci. 348, L75 (1996); Phys. Rev. Lett. 77, 1087 (1996).

${ }^{28}$ J. M. C. Thornton, A. A. Williams, J. E. Macdonald, R. G. van Silfhout, M. S. Finney, and C. Norris, Surf. Sci. 273, 1 (1992).

${ }^{29}$ H. J. Osten, J. Klatt, G. Lippert, E. Bugiel, and S. Hinrich, Appl. Phys. Lett. 60, 2522 (1992); H. J. Osten et al. Phys. Rev. Lett. 69, 450 (1992).

30 T. Ide, Phys. Rev. B 51, 5397 (1995).

${ }^{31}$ J. Nogami, A. A. Baski, and C. F. Quate, Appl. Phys. Lett. 58, 475 (1991).

${ }^{32}$ R. I. G. Uhrberg, R. D. Bringans, R. Z. Bachrach, and J. E. Northrup, Phys. Rev. Lett. 56, 520 (1986).

${ }^{33}$ R. S. Becker, T. Klitsner, and J. S. Vickers, J. Microsc. 152, 157 (1988).

${ }^{34}$ G. Ehrlich and F. G. Hudda, J. Chem. Phys. 44, 1039 (1966).

${ }^{35}$ R. L. Schwoebel and E. J. Shipsey, J. Appl. Phys. 37, 3682 (1966).

${ }^{36}$ P. Politi, G. Grenet, A. Marty, A. Ponchet, and J. Villain, Phys. Rep. 324, 271 (2000).

${ }^{37}$ Z. Zhang and M. Lagally, Phys. Rev. Lett. 72, 693 (1994).

${ }^{38}$ C. W. Oh, E. Kim, and Y. H. Lee, Phys. Rev. Lett. 76, 776 (1996).

${ }^{39}$ I. Markov, Phys. Rev. B 50, 11271 (1994)..

${ }^{40}$ I. Markov, Mater. Chem. Phys. 49, 93 (1997).

${ }^{41}$ J. A. Venables, G. D. T. Spiller, and M. Hanbücken, Rep. Progr. Phys. 47, 399 (1984).

42 S. Stoyanov and D. Kashchiev, in Current Topics in Materials Science, edited by E. Kaldis, (North-Holland, 1981), Vol. 7, p. 69.

${ }^{43}$ D. Kandel, Phys. Rev. Lett. 78, 499 (1997).

${ }^{44}$ I. Markov, Phys. Rev. B 56, 12544 (1997).

${ }^{45}$ S. Iwanari and K. Takayanagi, J. Cryst. Growth 119, 229 (1992).

${ }^{46}$ I.-S. Hwang, T.-C. Chang, and T. T. Tsong, Phys. Rev. Lett. 80, 4229 (1998).

${ }^{47}$ D. Kandel and E. Kaxiras, Phys. Rev. Lett. 75, 2742 (1995).

48 Young-Jo Ko, K. J. Chang, and Jae-Yel Yi, Phys. Rev. B 60, 1777 (1999).

${ }^{49}$ I. Markov, Phys. Rev. B 59, 1689 (1999).

${ }^{50}$ I. Markov, Surf. Sci. 429, 102 (1999).

${ }^{51}$ I. Markov, Phys. Rev. B 53, 4148 (1996).

${ }^{52}$ S. Stoyanov and I. Markov, Surf. Sci. 116, 313 (1982).

53 J. Tersoff, A. W. Denier van der Gon, and R. M. Tromp, Phys. Rev. Lett. 72, 266 (1994).

${ }^{54}$ J. Krug, P. Politi, and T. Michely, Phys. Rev. B 61, 14037 (2000).

55 S. Heinrichs, J. Rottler, and P. Maass, e-print cond/mat 0004031.

${ }^{56}$ S. Stoyanov, J. Cryst. Growth 94, 751 (1989).
${ }^{57}$ E. Kaxiras, Europhys. Lett. 21, 685 (1993).

${ }^{58}$ J. E. Griffith and G. P. Kochanski, Crit. Rev. Solid State Mater. Sci. 16, 255 (1990).

${ }^{59}$ U. Köhler, J. E. Demuth, and R. J. Hamers, J. Vac. Sci. Technol. A 7, 2860 (1989).

${ }^{60}$ B. Voigtländer, A. Zinner, T. Weber, and H. Bonzel, Phys. Rev. B 51, 7583 (1995).

${ }^{61}$ I.-S. Hwang, T.-C. Chang, and T. T. Tsong, Surf. Sci. 410, L741 (1998).

${ }^{62}$ B. Voigtländer and A. Zinner, J. Vac Sci. Technol. A 12, 1932 (1994).

${ }^{63}$ M. Katayama, T. Nakayama, M. Aono, and C. F. McConville, Phys. Rev. B 54, 8600 (1996).

${ }^{64}$ M. Copel, M. C. Reuter, M. Horn von Hoegen, and R. Tromp, Phys. Rev. B 42, 11682 (1990).

${ }^{65}$ F. K. LeGoues, M. Copel, and R. M. Tromp, Phys. Rev. Lett. 63, 1826 (1989).

${ }^{66}$ M. Horn-von Hoegen, A. Al-Falou, B. H. Müller, U. Köhler, L. Andersohn, B. Dahlmeier, and M. Henzler, Phys. Rev. B 49, 2637 (1994).

${ }^{67}$ M. Horn-von Hoegen, private communication.

${ }^{68}$ P. M. Marée, K. Nakagawa, F. M. Mulders, J. F. van der Veen, and K. L. Kavanagh, Surf. Sci. 191, 305 (1987).

FIG. 1. Schematic dependence of the film chemical potent- ial on the film thickness in number of monolayers for the three modes of growth denoted at each curve: VW Volmer-Weber, SK - Stranski-Krastanov, and FM - Frank-van der Merwe. 


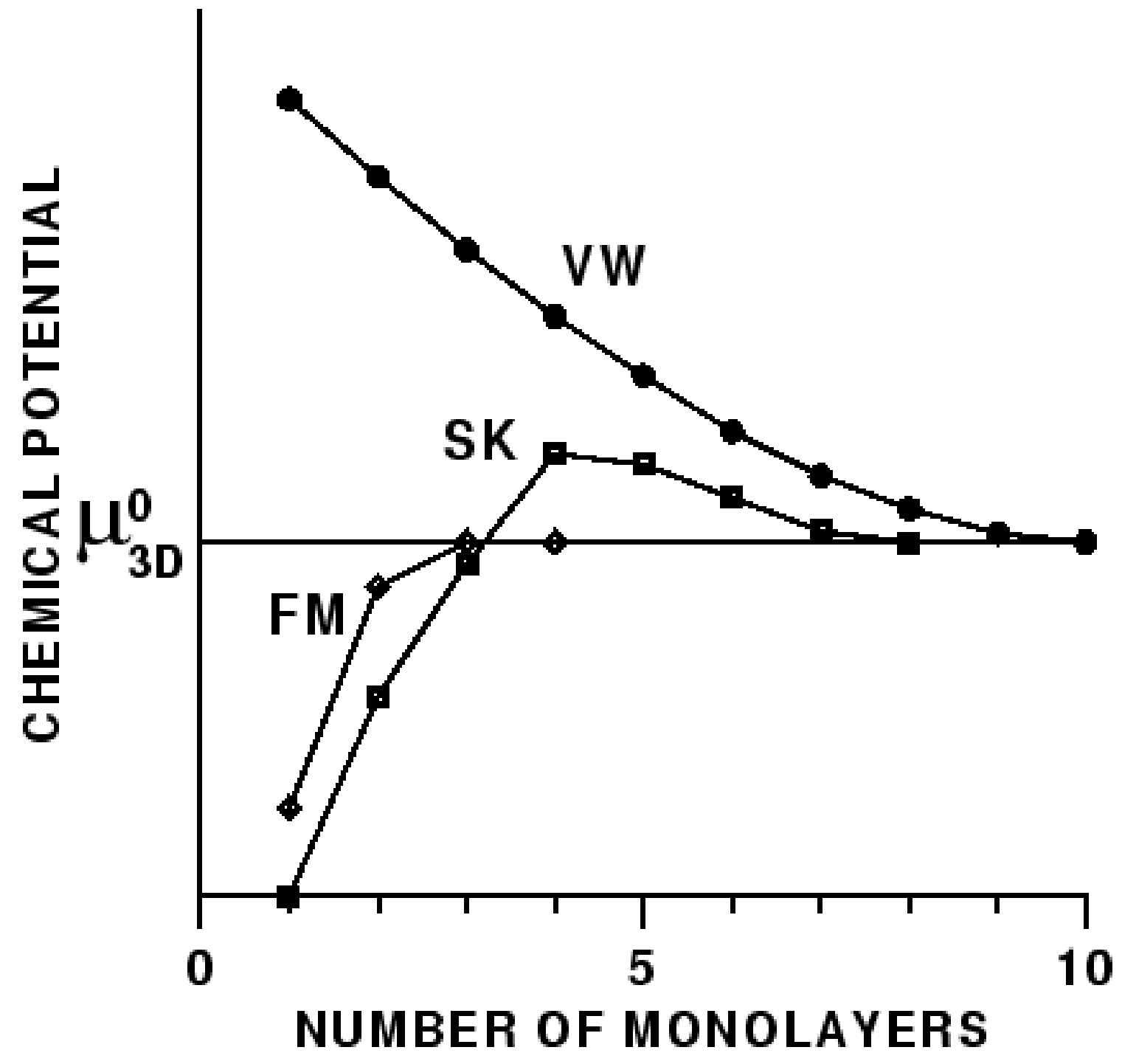

